\title{
IMINATION OF CHITOSAN NANOFIBERS \\ IN A HETEROGENEOUS SYSTEM. SYNTHESIS OPTIMIZATION AND IMPACT ON FIBER MORPHOLOGY
}

\author{
ALEXANDRU ANISIEI, ${ }^{*}$ ANDRA-CRISTINA BOSTANARU, ${ }^{* *}$ MIHAI MARES ${ }^{* *}$ \\ and LUMINITA MARIN ${ }^{*}$ \\ " "Petru Poni" Institute of Macromolecular Chemistry, Iasi, Romania \\ ** "Ion Ionescu de la Brad" University, Laboratory of Antimicrobial Chemotherapy, Iasi, Romania \\ ๔ Corresponding author: L. Marin,lmarin@icmpp.ro
}

Received June 4, 2021

The paper aimed to prepare imino-chitosan fibers by the imination reaction in a heterogenous system, targeting the improvement of anti-pathogenic activity. To this end, porous neat chitosan fibers were prepared by electrospinning of the polyethylene glycol/chitosan blend, followed by polyethylene glycol removal. Imination of the neat chitosan fibers was carried out in three liquid phase systems using solvents of different polarity and, consequently, different ability to swell the solid phase chitosan fibers. The successful imination was qualitatively and quantitatively assessed by FTIR and ${ }^{1} \mathrm{H}-\mathrm{NMR}$ spectroscopy, and the impact of the liquid phase on the fibers' morphology was evaluated by SEM, POM and AFM microscopy. Further, the antimicrobial activity of the imino-chitosan fibers was investigated on relevant bacterial and fungal strains. It was concluded that the prior swelling in water of the fibers improved the imination degree, while the use of a less polar solvent, such as toluene, favored the preservation of the fibrous morphology. The imination with an antimicrobial aldehyde endowed the chitosan fibers with the ability to create a physical barrier against pathogens.

Keywords: chitosan, imine, nanofibers, electrospinning

\section{INTRODUCTION}

Nanofibers are a class of materials with a large technological and commercial application in biomedicine (tissue engineering, drug delivery, and cancer diagnosis), food industry (coating materials), textiles, environment protection (air filtration) and electronics (optical sensors, lithium-air batteries). ${ }^{1-3}$ This is due to their high surface area-to-volume ratio, which generates a high active surface and, consequently, enhances their performance for many applications. The most commonly used method for nanofiber preparation is electrospinning, a versatile technique with a relatively high production rate and a simple setup, which allows transforming various polymers into fibrous mats. ${ }^{4,5}$ As different applications require specific properties, the chemical composition of the raw materials subjected to electrospinning is of utmost importance. In this regard, chitosan is of special interest for biomedicine due to its intrinsic properties, such as biocompatibility, biodegrada- bility, as well as antimicrobial, hemostatic and immune-enhancing effects. ${ }^{6}$ The manufacturing of chitosan nanofibers brings the advantage of good similitude with an extracellular matrix, making them suitable biomaterials for tissue engineering. Many research groups have dedicated their activity to the design of chitosan-based nanofibers as scaffolds for tissue engineering, pursuing strategies mainly directed to the encapsulation of bioactive agents, such as drugs, essential oils, natural extracts, metal nanoparticles, bioactive glass, hydroxyapatite and silica. ${ }^{7}$ This strategy gave notorious results, improving the bioactivity of chitosan fibers, while preserving their biocompatibility.

The advance of chemistry in the last decades has brought into researchers' attention the potential of dynamic covalent chemistry (DCC) as an important approach to creating materials that respond under the influence of external stimuli. ${ }^{8}$ Imine linkage formation is a powerful tool of 
DCC, due to its reversible nature, which allows easy exchange of components. ${ }^{9}$ Considering that chitosan is a polyamine, its imination with aldehydes should create valuable dynamic materials. This has been exploited for the preparation of chitosan hydrogels, drug delivery formulations or bioactive films, ${ }^{10-15}$ but less attention has been directed to imino-chitosan fibers. ${ }^{16}$ It has been demonstrated that the derivatization of chitosan by imination with aldehydes is a simple route to create multifunctional biomaterials.

Starting from these premises, the goal of paper was to prepare imino-chitosan fibers by the reaction of neat chitosan fibers with an aldehyde, in a heterogeneous system. The aldehyde has been chosen to contain an ortho-boronic acid moiety that can modulate the imine reversibility through intramolecular stabilization by $\mathrm{B}-\mathrm{N}$ and $\mathrm{H}$ bonds. ${ }^{17}$ Besides, the aldehyde has intrinsic antimicrobial activity. ${ }^{11}$ In such a way, dynamic biomaterials can be obtained, which can release the antimicrobial aldehyde in a controlled manner, by shifting the imination equilibrium under the influence of bacterial/fungal stimuli.

\section{EXPERIMENTAL}

\section{Materials}

Low molecular weight chitosan, with a molecular weight value calculated by viscosimetry of $195 \mathrm{kDa}$ and a degree of deacetylation of $85 \%$, PEO, with a molecular weight of $900 \mathrm{kDa}$, and 2formylphenylboronic acid (95\%) were purchased from Sigma-Aldrich. Glacial acetic acid was purchased from ChemReactiv, sodium hydroxide - from Fluka, toluene - from Carl Roth. Their use was performed as such, without any further purification.

\section{Preparation of chitosan nanofibers}

A chitosan/PEO mixture in a $7 / 3$ mass ratio was dissolved in $75 \%$ acetic acid to give a $4.5 \%(\mathrm{w} / \mathrm{v})$ solution. The solution was magnetically stirred at room temperature overnight in order to reach good homogenization. Further, the solution was transferred into a syringe with a stainless-steel blunt needle, with an inner diameter equal with $0.8 \mathrm{~mm}$, and the syringe was connected to an electrospinning device. Applying the following parameters: voltage $=25 \mathrm{kV}$, tip-tocollector distance $=12 \mathrm{~cm}$, flow $=0.6 \mathrm{~mL} / \mathrm{h}$, the electrospinning process proceeded smoothly, yielding a fiber mat. Further, the PEO co-electrospinning agent was removed by washing.

First, the chitosan/PEO fibers were immersed in a $5 \% \mathrm{NaOH}$ solution for 3 hours in order to remove the residual $\mathrm{AcOH}$ and then, they were immersed in distilled water (three times, for 5 hours each time), in order to remove $\mathrm{PEO}$ and eventually $\mathrm{NaOH}$ traces. The obtained fibrous mat was lyophilized at $0.01 \mathrm{mbar}$ to remove the water and to preserve the pores gained by PEO removal. The schematic representation of the procedure of chitosan nanofiber preparation is given in Scheme 1.

Functionalization of the chitosan fibers with 2formylphenylboronic acid

The as-obtained chitosan nanofibers were functionalized by the imination reaction with 2formylphenylboronic acid in a heterogeneous system, for a $1 / 1$ molar ratio of the glucosamine/aldehyde functional groups. Different solvents were used in order to optimize the reaction protocol towards a maximum reaction yield. Thus, the chitosan nanofibers were immersed into a $10 \%(\mathrm{w} / \mathrm{v})$ solution of 2formylphenylboronic acid, and the reaction vial was maintained at $50{ }^{\circ} \mathrm{C}$ for 24 hours, under slight magnetic stirring. Toluene and ethanol were used as solvents due to their ability to ensure good chitosan swelling, improving the access of aldehyde to the amine sites of chitosan. ${ }^{18}$

Moreover, in the case of toluene, the imination reaction was also performed on previously water swollen fibers.

After 24 hours, the fibers were removed from the reaction medium, washed with dry ethanol in order to remove the unreacted aldehyde, and dried at room temperature. The reaction conditions were schematically drawn in Scheme 2.

\section{Equipment and methods}

The freeze-drying was done with a $\mathrm{LABCONCO}$ FreeZone Freeze Dry System, at $-50{ }^{\circ} \mathrm{C}, 1.510 \mathrm{mbar}$, for 24 hours. The wet fiber mats were previously frozen in liquid nitrogen.

The FTIR spectra were recorded with a Bruker Vertex 70 FT-IR spectrophotometer, on $\mathrm{KBr}$ pellets prepared with the same amounts of milled samples. In order to estimate an average imination degree, five recordings were performed for each fiber sample. The imination degree was assessed using the absorbances (A) of the imine $\left(1640 \mathrm{~cm}^{-1}\right)$ and amine $\left(1543 \mathrm{~cm}^{-1}\right)$ bands, with the following equation:

$$
\text { CD }(\text { FTIR })=\frac{\mathrm{R}}{(R+1)} * 100
$$

where $\mathrm{R}=\mathrm{A}(-\mathrm{CH}=\mathrm{N}-) / \mathrm{A}(-\mathrm{NH} 2)$. The absorbance was measured with Bruker OPUS software, after the spectra baseline was realized. The absorbance values, their ratio and the resulted reaction yields $(\mathrm{CD}$ (FTIR)) were given in Table 1.

${ }^{1} \mathrm{H}-\mathrm{NMR}$ spectra were recorded on a Bruker Avance DRX $400 \mathrm{MHz}$ Spectrometer, at room temperature, with an accumulation of 128 scans. The fiber samples were completely dissolved in $2 \%$ acetic acid in $\mathrm{D}_{2} \mathrm{O}$, when the imination formation was completely shifted to the reagents. This allowed evaluating the conversion degree of the amine units of 
chitosan into imine units from the ratio of the integrated intensities of the chemical shift of aldehyde and $\mathrm{H} 2$ chitosan protons:

$\mathrm{CD}(\mathrm{NMR})=\frac{I(\mathrm{CHO})}{I(\mathrm{H} 2)+08 \mathrm{SS}} * 100$

where $\mathrm{I}(\mathrm{CHO})$ is the integral of the chemical shift of the aldehyde protons; $\mathrm{I}(\mathrm{H} 2)$ is the integral of the chemical shift of the $\mathrm{H} 2$ of chitosan and 0.85 is the deacetylation degree of chitosan.

Scanning electron microscopy was done with a field emission Scanning Electron Microscope SEM EDAX - Quanta 200, operated at an accelerating voltage of $20 \mathrm{keV}$. The average diameter of the fibers was measured using the Image J 1.52a software, as an average value of 100 measurements.

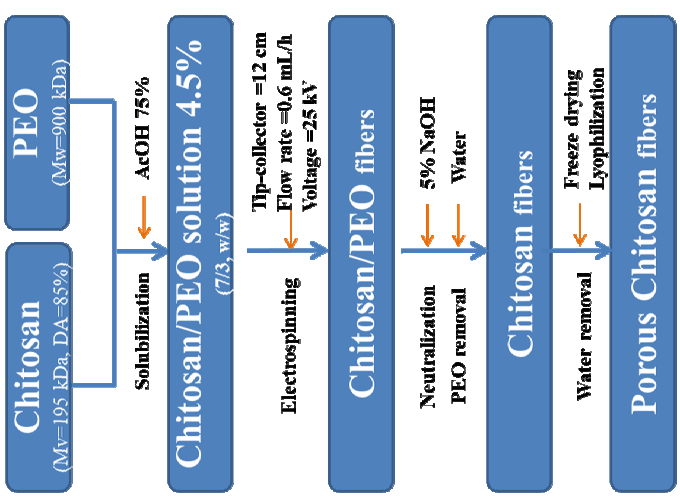

Scheme 1: Preparation of neat chitosan fibers

AFM measurements were performed on a commercial AFM NTMDT. The images were taken in the tapping mode with typical resonance frequency of

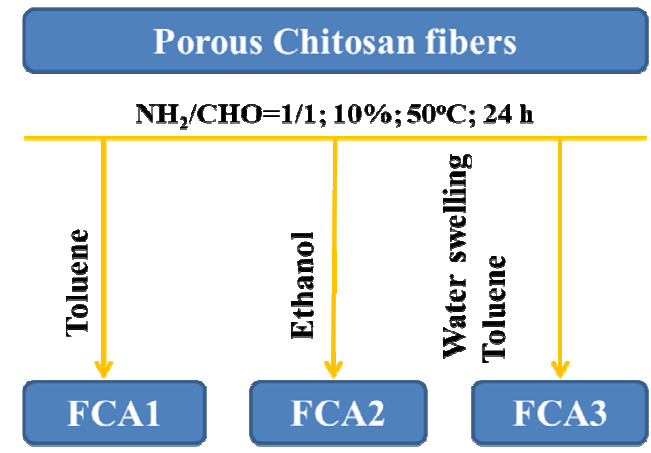

Scheme 2: Functionalization of chitosan fibers with 2-formylphenyl boronic acid

$240 \mathrm{kHz}$. Dry fibers were supported on glass with double-sided tape.

Table 1

Conversion degree of amine groups into imine units calculated from FTIR

\begin{tabular}{lccc}
\hline Code & FCA1 & FCA2 & FCA3 \\
\hline $\mathrm{A}(-\mathrm{NH} 2)$ & 0.143 & 0.123 & 0.118 \\
$\mathrm{~A}(-\mathrm{CH}=\mathrm{N}-)$ & 0.471 & 0.256 & 0.489 \\
$\mathrm{R}=\mathrm{A}(-\mathrm{CH}=\mathrm{N}-) / \mathrm{A}(-\mathrm{NH} 2)$ & 3.29 & 2.081 & 4.144 \\
$\mathrm{CD}(\mathrm{FTIR})$ & 76.6 & 67.5 & 80.5 \\
\hline
\end{tabular}

Table 2

Conversion degree of amine groups into imine units calculated from ${ }^{1} \mathrm{H}-\mathrm{NMR}$

\begin{tabular}{lccc}
\hline Code & FCA1 & FCA2 & FCA3 \\
\hline $\mathrm{I}(\mathrm{CHO})$ & 4.39 & 4.41 & 4.49 \\
$\mathrm{I}(\mathrm{H} 2)$ & 1.05 & 1.03 & 1.09 \\
$\mathrm{CD}(\mathrm{NMR})$ & 91.91 & 76.92 & 94.87 \\
\hline
\end{tabular}

The supramolecular organization of the chitosan chains during electrospinning was investigated by polarized optical microscopy (POM), performed with a Leica DM 2500 microscope.

The antimicrobial activity was investigated on three reference strains: Pseudomonas aeruginosa, methicillin-resistant Staphylococcus aureus and Candida albicans ATCC 10231. The microorganisms were stored at $-80{ }^{\circ} \mathrm{C}$ in $10 \%$ glycerol. All bacteria were refreshed in BHI broth (Bio-Rad, France) at 36
${ }^{\circ} \mathrm{C}$, and afterward were inoculated on Mueller-Hinton plates (Bio-Rad, France). The fungi were refreshed on Sabouraud Dextrose Broth (Bio-Rad, France) and were grown at $36{ }^{\circ} \mathrm{C}, \mathrm{pH}=7$. These cultures were further prepared as microbial suspensions in sterile saline solution to obtain an optically comparable turbidity to that of the 0.5 McFarland standards, yielding a suspension containing approximately $1 \times 10^{8} \mathrm{CFU} \mathrm{mL}$ 1. Volumes of $0.2 \mathrm{~mL}$ from each inoculum were spread onto Mueller-Hinton Agar pre-poured in Petri dishes 
and when the medium surface was dried, the samples were added. The fibrous samples were previously prepared as round pellets with a hydraulic press at 2 $\mathrm{N} / \mathrm{m}^{2}$. To evaluate the antimicrobial properties, the inhibition of growth was measured under standard conditions after $24 \mathrm{~h}$ of incubation at $36{ }^{\circ} \mathrm{C}$. The Petri dishes were observed under a stereomicroscope and the images were processed to observe the co-growth of the colonies. Subsequently, in-depth observation of microfungal structures was performed through an optical microscope, at magnification of $40 x$ and $63 \times$. The tests were carried out in triplicate. After measurements, the samples were inactivated at $121{ }^{\circ} \mathrm{C}$ for 20 minutes and analyzed for surface and structure modifications.

\section{RESULTS AND DISCUSSION}

Synthesis and structural characterization

With the aim to prepare biomaterials with antimicrobial properties, chitosan fibers were electrospun and further functionalized by the imination reaction with an aldehyde that proved anti-microbial activity.

The imination reaction was realized in a heterogeneous system, by immersing the chitosan fibers into an aldehyde solution. In order to find the optimal imination conditions for a high reaction yield, solvents of different polarity were used for the preparation of the liquid phase, such as (i) ethanol, (ii) toluene, or (iii) the were fibers priorly swollen in water and then immersed in a toluene solution (Scheme 2). The solvents were chosen in order to assure: (i) swelling of the chitosan fibers, and thus the availability of amine functional groups, and (ii) good aldehyde dissolution and thus good mobility of its molecules, enhancing consequently the number of effective collisions towards a higher imination yield.

The successful imination in a heterogeneous system was firstly assessed by FTIR spectroscopy. As can be seen in Figure 1, a sharp absorption band at $1640 \mathrm{~cm}^{-1}$ appeared for all functionalized samples, confirming the condensation reaction between the amine groups of chitosan and 2-formylphenylboronic acid. ${ }^{11}$ The intensity of the imine band increased once the intensity of the absorption band characteristic of the amine units $\left(1543 \mathrm{~cm}^{-1}\right)$ decreased. No absorption band characteristic of the vibration of the aldehyde functional group was detected. Besides, the vibration of the boric acid residue and the double linkage of the aromatic ring were detected at 764 and $1462 \mathrm{~cm}^{-1}$, respectively. These data suggested that all the reaction protocols yielded imino-chitosan derivatives. A rough evaluation of the imination degree from the absorbances ratio of imine and amine bands ${ }^{19}$ indicated reaction yields higher than 67\%. It appeared that the previous swelling in water of the fibers offered the best conditions for an efficient imination reaction, even though the water supports the shifting of the imination equilibrium to the reagents. ${ }^{20}$ This can be attributed to the ability of water to form an azeotropic mixture with toluene, pushing the imination equilibrium to the products.

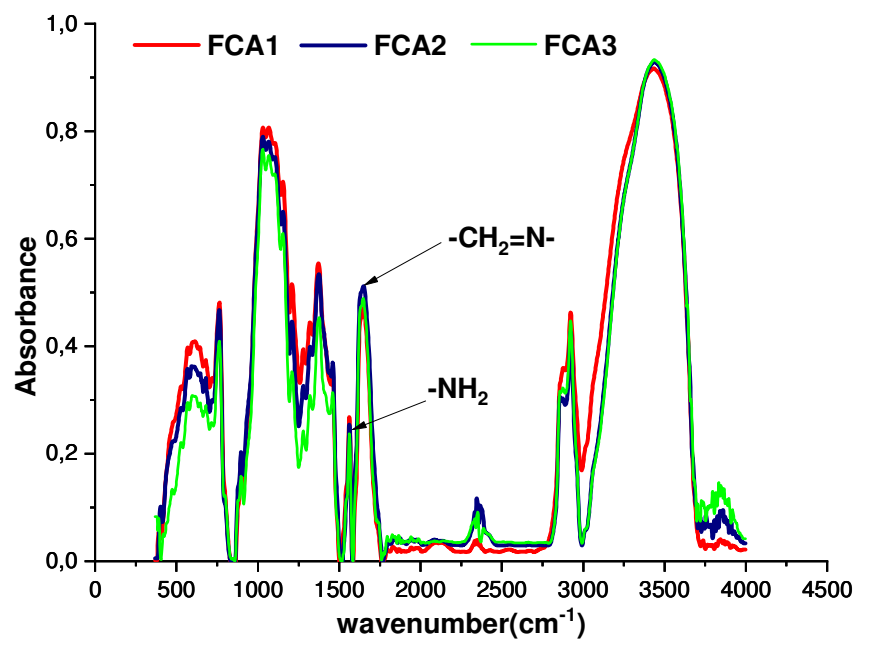

Figure 1: FTIR spectra of functionalized fibers 


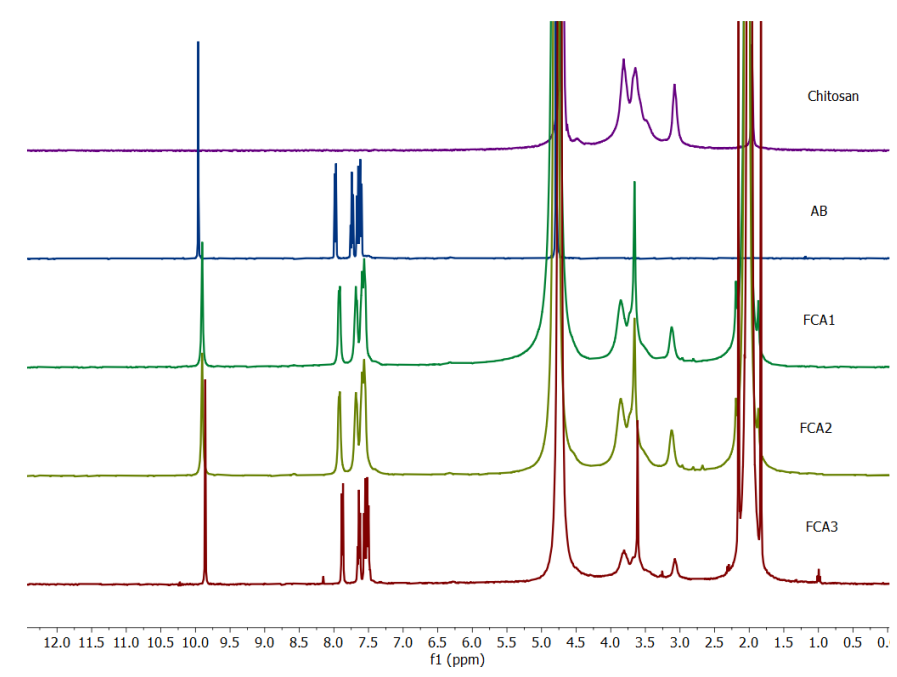

Figure 2: ${ }^{1} \mathrm{H}-\mathrm{NMR}$ spectra of the studied chitosan derivatives (FCA1, FCA1 and FCA3), as well as chitosan and aldehyde $(\mathrm{AB})$ references

Further, a complementary evaluation of the imination degree was realized by ${ }^{1} \mathrm{H}-\mathrm{NMR}$ spectroscopy. The total dissolution of the functionalized fibers was realized in $2 \%$ acetic acid in deuterated water, which inflicted total shifting of the imination reaction to the reagents, as confirmed by the presence of the aldehyde proton at $9.9 \mathrm{ppm}$, and the total absence of the imine proton $(8.6 \mathrm{ppm}) .{ }^{11}$ The chemical shift of aromatic protons were also present, at 7.4 and 7.8 $\mathrm{ppm}$, in the right ratio of their integrals, suggesting that no side reactions occurred, but just the shifting of the imination equilibrium to the reagents.

A more precise quantitative insight on the reaction pathway was estimated from the ratio between the signal area of imine and $\mathrm{H} 2$ of chitosan. ${ }^{11}$ The chemical shift of the $\mathrm{H} 2$ peak at $3.12 \mathrm{ppm}$ has been chosen as reference for glucosamine units, because its shape was not superposed with other signals, and thus, its area was not affected. ${ }^{21}$ Applying Equation 2, the conversion degree of the amine groups of chitosan into imine bonds gave values higher than $76 \%$ (Table 2). Even if the conversion degree values obtained from NMR were almost $15 \%$ higher than those from FTIR, they maintained the same trend, indicating the fibers' swelling in water as the most suitable route for a high imination yield. The differences between the conversion degree values obtained by the two methods can be explained by the difficulty to extract the absorbance of the amide units that superposed with that of the imine ones in the FTIR spectra.

\section{Morphology}

To estimate the impact of the imination route on fiber morphology, SEM images of the functionalized fibers were acquired and compared to those of neat chitosan fibers (Fig. 3). The SEM images of the electrospun CS/PEO sample showed long fibers, with no beads and an average diameter of $276 \mathrm{~nm}$ (Fig. 3a). PEO removal in water appeared to cause fiber entanglement, most probably because of polar water molecules, which favored new $\mathrm{H}$-bonds between chitosan in neighbor fibers (Fig. 3b). This morphology was preserved for the FCA1 fibers functionalized in non-polar toluene (Fig. 3c). On the other hand, the FCA2 fibers functionalized in the polar ethanol showed a strong coalescence of the fibers into a more compact biomaterial with the aspect of a porous film (Fig. 3d). A similar behavior was evident for the fibers swollen in water before the imination reaction in toluene (Fig. 3e).

It was expected that the removal of PEO from the fibers would lead to a porous inner morphology, which is favorable to the imination reaction. To explore this hypothesis, the topography of CS/PEO and CS fibers was investigated by atomic force microscopy (Fig. 4). First, AFM images confirmed the fiber entanglement after PEO removal, by an increase in the average diameter of the fibers and a decrease in the inter-fiber pores, from 1-2 $\mu \mathrm{m}$ to less than $1 \mu \mathrm{m}$ (Fig. 4a, c). This was in line with the preservation of the swollen state during the fast freezing in nitrogen and lyophilization. The magnification at the nanometric level of the fiber 
revealed drastic changes in fiber topography. It appeared that, during PEO removal, the fiber surface became rougher, with the appearance of channels, suggesting the occurrence of long interfiber pores (Fig. 4b, d).

Another important aspect related to fiber morphology is fiber crystallinity. It is known that

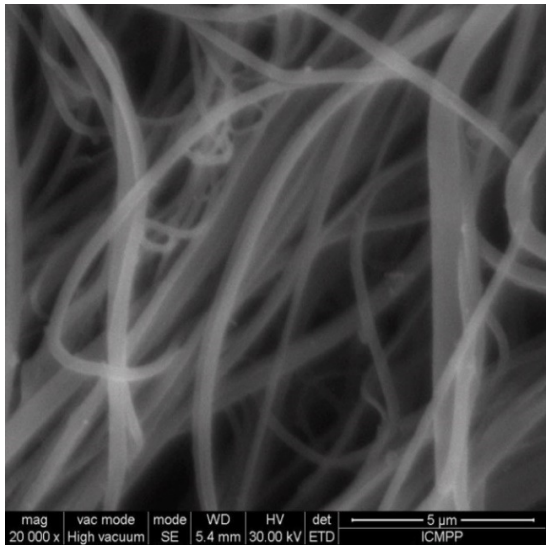

a) $\mathrm{CS} / \mathrm{PEO}$

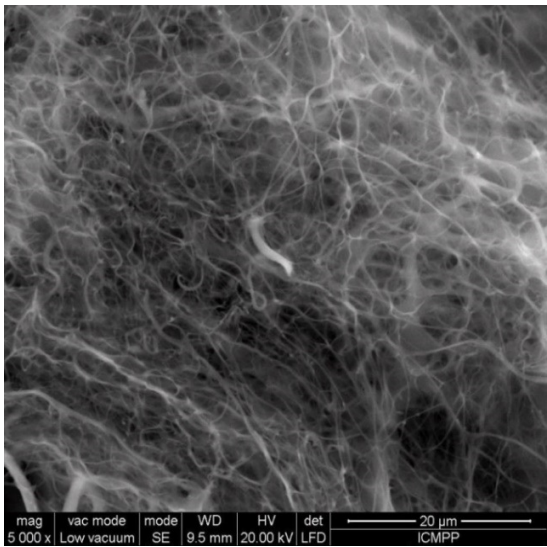

c) CS

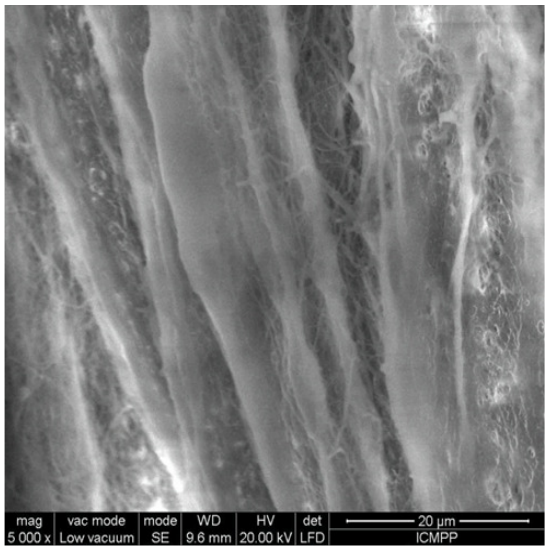

e) FCA2 a higher crystallinity degree induces higher mechanical properties of the fibers. ${ }^{22} \mathrm{~A}$ rough estimation of fiber crystallinity was done by polarized light microscopy. The CS/PEO and CS fiber mats showed birefringent textures under polarized light, indicating the alignment of the chitosan chains during electrospinning.

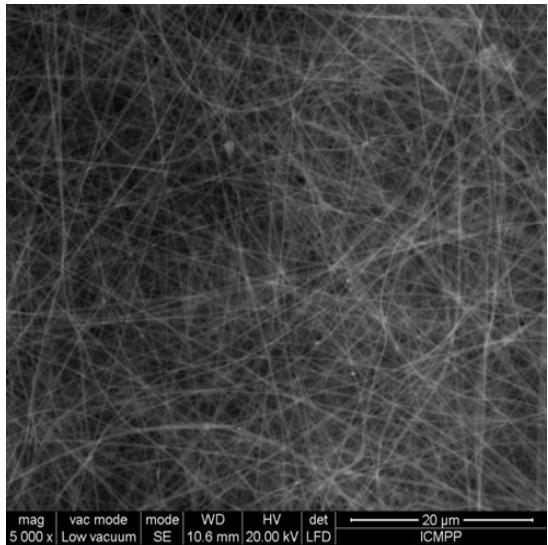

b) CS/PEO

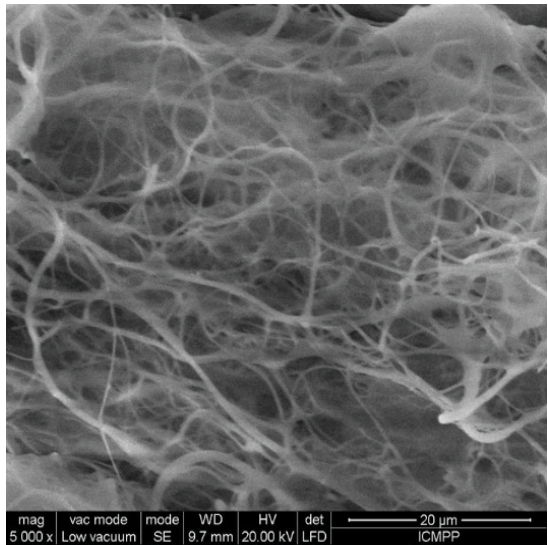

d) FCA1

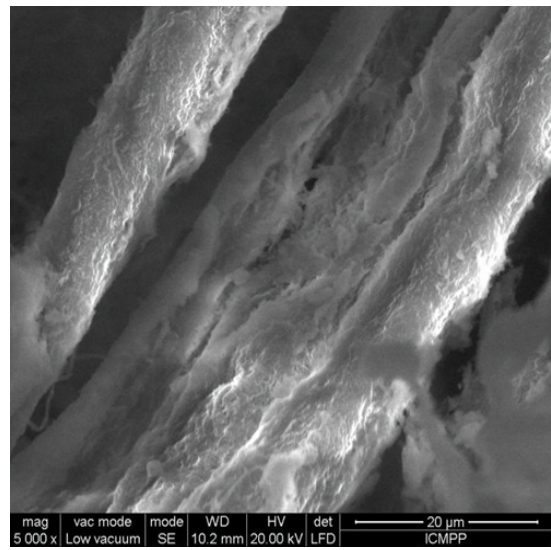

f) FCA3

Figure 3: SEM images of the studied fibers 

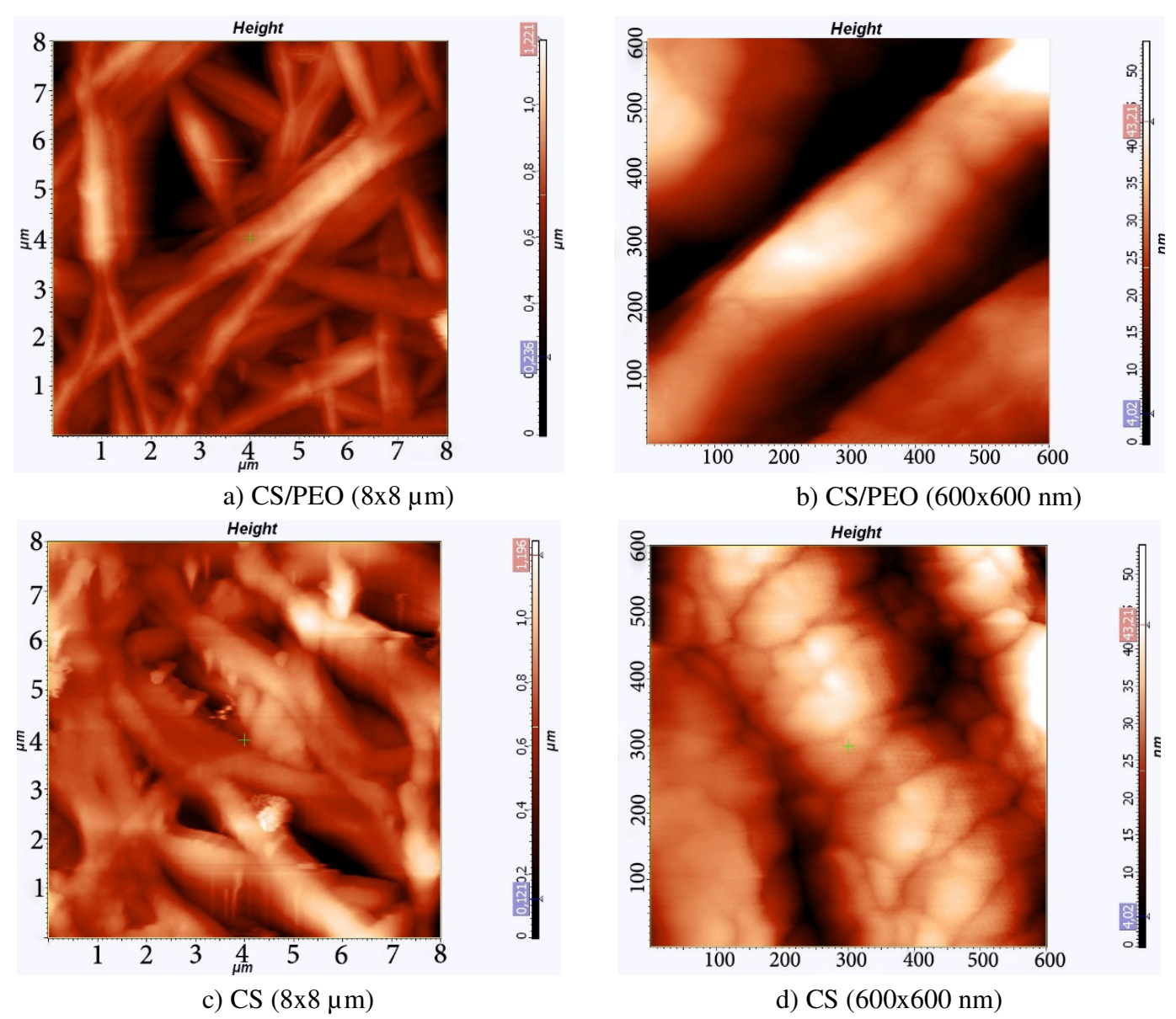

b) $\mathrm{CS} / \mathrm{PEO}(600 \times 600 \mathrm{~nm})$
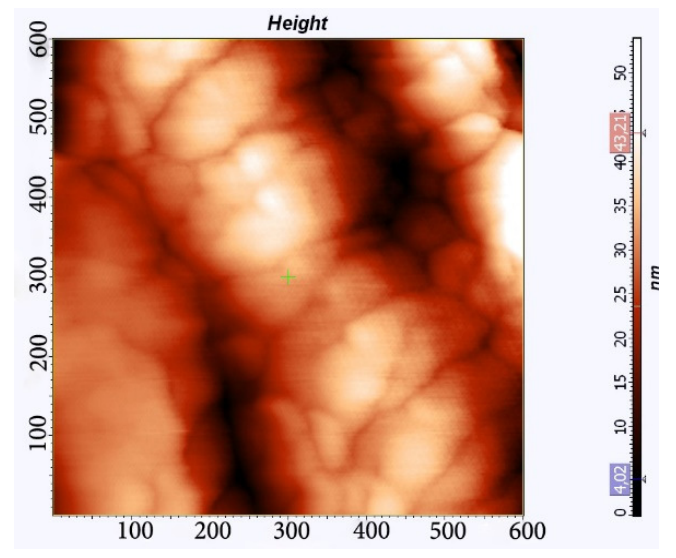

Figure 4: AFM images of CS-PEO (a, b) and CS (c, d) fibers

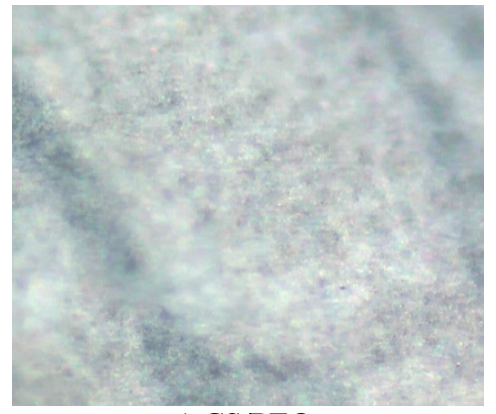

a) $\mathrm{CS} / \mathrm{PEO}$

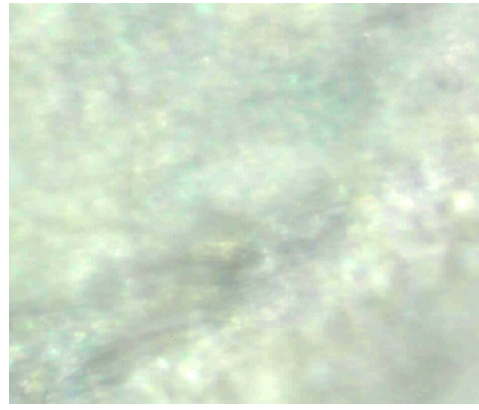

b) CS

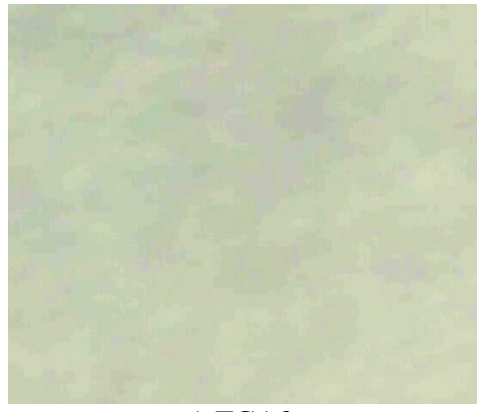

c) FCA3

Figure 5: Representative POM images of the studied fibers

The birefringence was not lost by imination, indicating the preservation of the crystallinity degree (Fig. 5). This is an important aspect for the prospective application of the fibers for wound healing applications, which require biomaterials with good mechanical properties. ${ }^{23}$

\section{Antimicrobial activity}

The activity of imino-chitosan nanofibers was tested against relevant gram-positive (methicillin- resistant Staphylococcus aureus) and gramnegative (Pseudomonas aeruginosa) bacteria and Candida albicans fungus (Fig. 6). All the fibers inhibited the strains' growth in their proximity, with clear inhibition zones against methicillinresistant Staphylococcus aureus, staph bacteria resistant to many antibiotics, easily spread in skin and soft tissue infections. ${ }^{24}$ This behavior is in line with the reversibility of imine linkage, which favors the release of new amounts of 
antimicrobial aldehyde once it is consumed in the inhibition process. The lack of higher activity was attributed to the dry medium for the in vitro experiment, which was not favorable for the

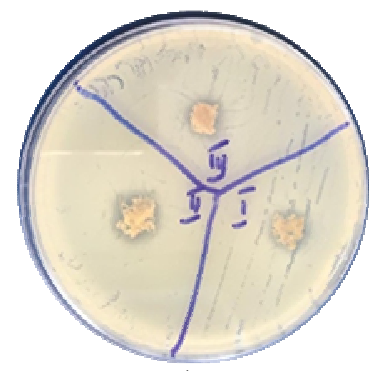

a)

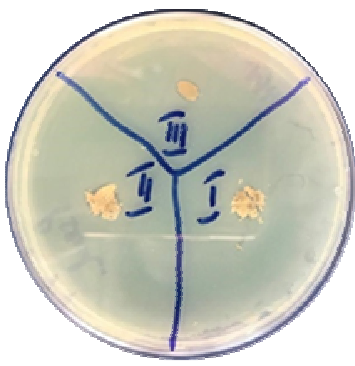

b)

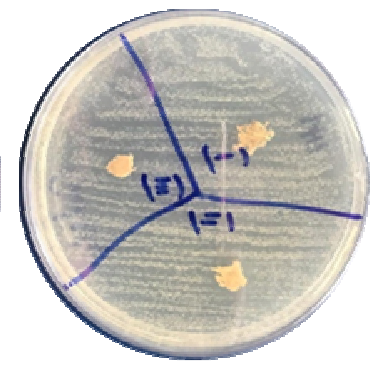

c)

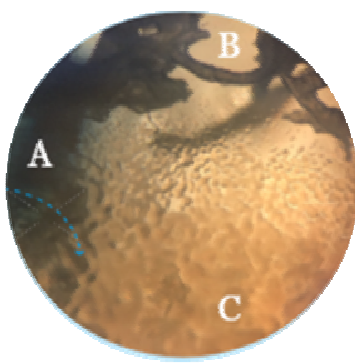

d)

Figure 6: Antimicrobial activity against: a) methicillin-resistant Staphylococcus aureus,

b) Pseudomonas aeruginosa and c) Candida albicans; d) Microscopic image of sample a) with: A: imino-chitosan fiber samples, B: inhibition zone, C: confluent colonies of the tested strain

\section{CONCLUSION}

Imino-chitosan fibers were prepared by heterogenous imination of porous neat chitosan fibers with an anti-pathogenic aldehyde in a heterogenous system. The conversion degree of the amino groups of chitosan into imine units varied as a function of the polarity of the solvent used to prepare the liquid phase (water, ethanol, toluene), the highest reaction yield being reached when the fibers were previously swollen in water. On the other hand, the polar water and ethanol solvents caused entanglement of the fibers towards a more compact biomaterial, while the less polar toluene preserved well the fiber morphology. The fibers presented antimicrobial activity, especially against methicillin-resistant Staphylococcus aureus. It was concluded that the imination of chitosan fibers is an accessible synthetic route for the preparation of biomaterials with improved properties for bioapplication.

ACKNOWLEDGEMENTS: This work was supported by a grant of the Ministry of Research, Innovation and Digitization, CNCS/CCCDIUEFISCDI, project number 538PED/2020 within PNCDI III.

\section{REFERENCES}

1 M. Alazab, G. R. Mitchell, F. J. Davis and S. D. Mohan, Proc. Manufact., 12, 66 (2017), https://doi.org/10.1016/j.promfg.2017.08.009

2 S. Chen, M. Wu, C. Wang, S. Yan, P. Lu et al., Polymers, 12, 1780 (2020), https://doi.org/10.3390/polym 12081780
3 R. E. Abouzeid, A. Salama, Z. A. Al-Ahmed, N. S. Awwad and M. A. Youssef, Cellulose Chem. Technol., 54, 237 (2020), https://doi.org/10.35812/CelluloseChemTechnol.2020. 54.25

4 N. Tucker, J. J. Stanger, M. P. Staiger, H. Razzaq and K. Hofman, J. Eng. Fiber Fabr., 7, 63 (2012), https://doi.org/10.1177/155892501200702S10

5 S. Ramakrishna, K. Fujihara, W. E. Teo, T. Yong, Z. Ma et al., Mater. Today, 9, 40 (2006), https://doi.org/10.1016/S1369-7021(06)71389-X

6 Y. Wu, A. Rashidpour, M. P. Almajano and I. Metón, Polymers, 12, $1177 \quad$ (2020), https://doi.org/10.3390/polym12051177

7 A. Anisiei, F. Oancea and L. Marin, Rev. Chem. Eng., (2021), https://doi.org/10.1515/revce-2021-0003

8 D. Su, Y. Zhang, S. Ulrich and M. Barboiu, ChemPlusChem, $\quad \mathbf{8 6}, \quad 1 \quad$ (2021), https://doi.org/10.1039/C9QM00598F

9 S. Kulchat, M. N. Chaur and J. M. Lehn, Chem. Eur. J., 23, $11108 \quad$ (2017), https://doi.org/10.1002/chem.201702088

10 R. Yu, L. Cornette de Saint-Cyr, L. Soussan, M. Barboiu and S. Li, Int. J. Biol. Macromol., 167, 1146 (2021), https://doi.org/10.3390/md19070359

11 L. Marin, D. Ailincai, M. Mares, E. Paslaru, M. Cristea et al., Carbohyd. Polym., 117, 762 (2015), https://doi.org/10.1016/j.carbpol.2014.10.050

12 A. Bejan, F. Doroftei, X. Cheng and L. Marin, Int. J. Biol. Macromol., 162, 1839 (2020), https://doi.org/10.1016/j.ijbiomac.2020.07.232

13 D. Ailincai, W. Porzio and L. Marin, Polymers, 12, 2687 (2020), https://doi.org/10.3390/polym12112687

14 I. A. Duceac, L. Verestiuc, C. D. Dimitriu, V. Maier and S. Coseri, Polymers, 12, 1473 (2020), https://doi.org/10.3390/polym12071473

15 S. Xiong, L. Duan and X. Cheng, Polym. Chem., 11, 6066 (2020), https://doi.org/10.1039/D0PY00802H 
16 L. Marin, B. Dragoi, N. Olaru, E. Perju, A. Coroaba et al., Eur. Polym. J., 120, 109214 (2019), https://doi.org/10.1016/j.eurpolymj.2019.109214

17 H. Chen, H. Ye, Y. Hai, L. Zhang and L. You, Chem. Sci., 11, 2707 (2020), https://doi.org/10.1039/C9SC05698J

18 L. Liu, Y. Wang, X. Shen and Y. Fang, Biopolymers, $\quad \mathbf{7 8 ,} \quad 163 \quad$ (2005), https://doi.org/10.1002/bip.20261

19 R. Varma and S. Vasudevan, ACS Omega, 5, 20224 (2020), https://doi.org/10.1021/acsomega.0c01903

20 V. Sagiomo and U. Luning, Tetrahedron Lett., 50, 4663

https://doi.org/10.1016/j.tetlet.2009.05.117
21 A. Hirai, H. Odani and A. Nakajima Polym. Bull., 26, 87 (1991), https://doi.org/10.1007/BF00299352

${ }^{22}$ H. Lee, K. Yamaguchi, T. Nagaishi, M. Murai, M. Kim et al., RSC Adv., 7, 43994 (2017), https://doi.org/10.1039/C7RA06545K

${ }_{23}$ B. Azimi, H. Maleki, L. Zavagna, J. G. De la Ossa, S. Linari et al., J. Funct. Biomater., 11, 67 (2020), https://doi.org/10.1039/C7RA06545K

24 J. P. Hoffmann, J. K. Friedman, Y. Wang, J. B. McLachlan, M. C. Sammarco et al., Front. Microbiol., 23, 3106

(2020),

https://doi.org/10.3389/fmicb.2019.03106

25 M. Spear, Plast. Surg. Nurs., 32, 77 (2012), https://doi.org/10.1097/PSN.0b013e318256d638 